\title{
INFLUÊNCIA DA DIETA HOSPITALAR NO ESTADO NUTRICIONAL DE ADULTOS INTERNADOS NO SERVIÇO DE TRANSPLANTE HEPÁTICO
}

\author{
The influence of nutritional intake on nutritional status of adults admitted to Liver Transplant Service
}

Francisca E Zaina', Reginaldo W Lopes², Layza Tiemann³, Alessandra Mello³

\begin{abstract}
RESUMO
Objetivo: Determinar a influência da ingestão dietética hospitalar sobre o estado nutricional de adultos candidatos ou submetidos a transplante hepático. Pacientes e métodos: estudo prospectivo avaliou 48 adultos, 81,3\% pré-transplante (idade média de 46,36 anos) e 18,7\% pós-transplante (idade média de 51,89 anos) internados no Serviço de Transplante Hepático do HC/UFPR, na data da internação e da alta. Foi aplicado o teste de T Student. Analisou-se sexo, idade, índice de massa corporal - adequação: do peso atual em relação ao peso usual e ao ideal, da reserva adiposa localizada e generalizada, da circunferência muscular do braço e controle da ingestão dietética/ necessidades nutricionais. Resultados: Para o índice de massa corporal, 51,28\% dos pacientes pré-transplante encontravam-se eutróficos na data da internação e da alta, enquanto que no pós-transplante $44,44 \%$ dos pacientes encontravam-se com excesso de peso no período da internação. Ocorreu algum grau de depleção adiposa localizada para os pré-transplantes, sendo 58,96\% no período da internação e $61,52 \%$ no período da alta; para os pós-transplantes a depleção foi de $44,44 \%$ na internação e $66,66 \%$ na alta; $95,83 \%$ não ingeriram as necessidades energéticas e $85,41 \%$ as necessidades protéicas. Houve diferença estatística $(p<0,05)$ para o percentual de adequação do peso ideal $(p=0,01)$ nos pré-transplantes e no percentual de adequação da gordura localizada $(p=0,03)$ para os pós-transplantes. Conclusão: as necessidades nutricionais podem ter sido superestimadas.
\end{abstract}

Descritores: Avaliação nutricional, Estado nutricional, Influência, Ingestão nutricional, Transplante hepático.

\footnotetext{
Trabalho realizado no serviço de Transplante Hepático do Hospital de Clínicas da UFPR

1. Nutricionista do Serviço de Transplante Hepático do HC/UFPR. Especialista em Nutrição Clínica;

2. Hepatologista, Professor Sênior do Departamento de Clínica Médica - UFPR;

3. Acadêmica do curso de nutrição da UTP e da UFPR, respectivamente.

Endereço para correspondência: Francisca Eugênia Zaina - Rua General Carneiro, 180 (UND) Curitiba - PR - CEP 80060-900 - Fone: (41) 3324-6189 - 3360-1892 E-mail: fezaina@hotmail.com

Recebido em: 30/06/2003

Aceito em: 21/09/2004
}

\section{INTRODUÇÃO}

Em 1967, houve o $1^{\circ}$ resultado favorável do transplante hepático (TH), tornando-o uma realidade. ${ }^{1}$ Inúmeros fatores interferem no resultado pós-operatório, entre eles o estado nutricional. Sendo a desnutrição um fator de risco, o tempo de sobrevida dos pacientes submetidos ao TH é influenciado. ${ }^{2}$ A desnutrição é freqüente em pacientes hospitalizados ${ }^{3}$ e está associada com uma maior permanência hospitalar, menor resistência à infecção, cicatrização de feridas diminuída e maior morbimortalidade. ${ }^{4} \mathrm{~A}$ incidência de desnutrição aumenta conforme se aumenta o tempo de hospitalização. ${ }^{5}$ Os pacientes hepáticos são na maioria das vezes desnutridos. ${ }^{6}$ Os fatores envolvidos no mecanismo de desnutrição são principalmente: inadequada ingestão dietética, má absorção de nutrientes, aumento de requerimento energético e anormalidades metabólicas extra hepáticas conduzindo para aceleração do estado de inanição. ${ }^{78}$ A gravidade da falência hepática é provavelmente a principal causa de um suporte nutricional ineficaz, pois metabolismo protéico e energético dependem da função hepática. ${ }^{9}$

\section{PACIENTES E MÉTODOS}

Estudo prospectivo, realizado entre novembro de 2002 e março de 2003, avaliou a influência da ingestão dietética hospitalar sobre 
o estado nutricional de 48 adultos internados no Serviço de Transplante Hepático (STH) do Hospital de Clínicas da Universidade Federal do Paraná, sendo $81,25 \%$ dos pacientes candidatos ao transplante hepático (pré-TH) $(7,7 \%$ child A, $64,1 \%$ B, e $28,2 \%$ C) e $18,75 \%$ dos pacientes submetidos ao transplante hepático (pós-TH) .Todos os pacientes tiveram como critério de inclusão: diagnóstico de doença hepática avançada, realização ou não do transplante, estado geral que permitisse a avaliação nutricional. Para pacientes que apresentavam ascite, foi considerado o peso seco para realização dos cálculos. A média de dias de controle da ingestão alimentar dos pacientes pré-TH foi de 4 dias, e dos pacientes pós-TH de 11,5 dias. Foram considerados como dados clínicos: sexo, idade e como dados nutricionais: índice de massa corporal-IMC, ${ }^{10}$ adequação do peso atual em relação ao peso usual-\%PA/PU, ${ }^{11}$ adequação do peso atual em relação do peso ideal \%PA/PI, ${ }^{11}$ reserva adiposa localizada-\% $\%$ PCT, ${ }^{12}$ reserva adiposa generalizada $\% \mathrm{G},{ }^{12}$ circunferência muscular do braço-\% $\% \mathrm{CMB},{ }^{12}$ e controle de ingestão dietética segundo protocolo de Nutrição do STH. Utilizou-se para análise estatística o método de Teste $\mathrm{T}$ Students $(\mathrm{p}<0,05)$, média e desvio padrão.

\section{RESULTADOS}

Para o grupo pré TH o diagnóstico predominante foi o vírus da

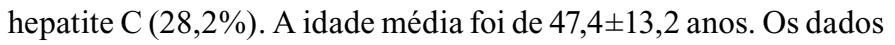
antropométricos são apresentados na tabela 1.

Verificou-se que a porcentagem da amostra que não ingeriu as necessidades energéticas $(95,83 \%)$ e protéicas $(85,4 \%)$ foi grande, mesmo alguns utilizando nutrição enteral via oral $(23,43$ $\%$ ). Dos pacientes avaliados, $66,7 \%$ tiveram a ingestão dietética afetada por intercorrências como jejum e não aceitação da dieta. A dieta mais freqüente foi a hipossódica: para o pré TH $(74,3 \%)$ e para o pós TH $(33,4 \%)$.

Encontrou-se diferença estatística para o \% PA/PI $(p=0,01)$ - grupo pré TH e para o \% PCT $(p=0,03)$ - grupo pós TH.
Tabela 1: Resultados antropométricos obtidos

\begin{tabular}{|c|c|c|c|c|}
\hline & \multicolumn{2}{|c|}{$\begin{array}{l}\text { Pré TH } \\
\mathrm{N}=39\end{array}$} & \multicolumn{2}{|c|}{ 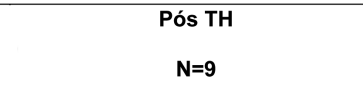 } \\
\hline & Internação & Alta & Internação & Alta \\
\hline$\% \mathrm{~Pa} / \mathrm{Pi}$ & $113,9 \pm 17,8$ & $111,8 \pm 17,5$ & $108,7 \pm 25,2$ & $104,7 \pm 26,2$ \\
\hline$\% \mathrm{~Pa} / \mathrm{Pu}$ & $91,5 \pm 12,5$ & $90,6 \pm 11,8$ & $94 \pm 13$ & $90,7 \pm 15,6$ \\
\hline IMC & $24,6 \pm 3,8$ & $24,4 \pm 3,8$ & $23,9 \pm 5,5$ & $23,3 \pm 5,5$ \\
\hline$\% \mathrm{PCT}$ & $76,3(16,6-212,5)$ & $74(16,6-216,6)$ & $104,1(29,1-131,8)$ & $86,3(29,1-104,1)$ \\
\hline$\% G$ & $24,8 \pm 8,4$ & $24,2 \pm 9$ & $25,2 \pm 11,2$ & $24 \pm 9,9$ \\
\hline$\%$ CMB & $92 \pm 12,4$ & $92,2 \pm 13$ & $94,1 \pm 16,2$ & $97,4 \pm 21,8$ \\
\hline
\end{tabular}

\section{DISCUSSÃO}

$\mathrm{O}$ diagnóstico mais comum foi a Cirrose por Vírus da Hepatite $\mathrm{C}$ (28,2\%), concordando com a literatura: ${ }^{13}$ já a cirrose alcoólica teve uma incidência $(20,51 \%)$ acima do relatado. ${ }^{14}$ Tanto na internação quanto na alta, prevaleceu a quantidade de pacientes eutróficos (51,28\%) para o pré TH . Para o pós TH foi a obesidade na internação $(44,44$ \%) e a eutrofia na alta (44,44\%); ao contrário de diversos estudos, que relatam que pacientes hepáticos são na maioria das vezes desnutridos. ${ }^{6,7,9,11}$ Verificou-se que no grupo pós TH, o número de pacientes abaixo do peso manteve-se, fato esse que não condiz com a literatura, já que ocorrem, na internação, fatores que promovem a desnutrição. ${ }^{15}$

\section{CONCLUSÃO}

A ingestão dietética inadequada não se refletiu em estado nutricional deficiente em períodos de até 4 dias para o pré TH e de 11,5 para o pós $\mathrm{TH}$, para a maioria dos padrões nutricionais avaliados entretanto as necessidades nutricionais calculadas de acordo com o protocolo de TH de nossa unidade, referendadas pela literatura vigente, podem ter sido superestimadas.

\section{SUMMARY}

Objective: To determine the influence of dietetic intake on nutricional status in adult candidates to orthotopic liver transplant (pre-transplant) and after the procedure (post-transplant). Methods: this prospective study evaluated 48 adults (81.3\%) pre-transplant with a mean age of 46.36 years and $18.7 \%$ of transplanted ones, with a mean age of 51.89 years, on admission in the hospital and on the day of discharge. "T" Student test was adopted. The variables studied were gender, age, body mass index, adequacy of current body weight for usual body weight and for ideal body weight, tricipital skin-fold, mid-upper arm muscle circumference, $\%$ generalized adipose reserve and nutritional intake control vs. nutritional requirements. Results: In terms of body mass index $51.28 \%$ of pre- transplant patients were eutrophic both on admission and discharge and $44.44 \%$ of post-transplant patients were overweight on admission and $44.44 \%$ were eutrophic on discharge. Some degree of depletion for adequacy of tricipital skin-fold occurred in pre-transplant in patients in 58.96\% of in patients and $61.52 \%$ on discharge and $44.44 \%$ on admission and $66.66 \%$ on discharge for Post-transplant patients. Concerning adequacy of mid-upper arm muscle circumference there was not significant incidence of depletion in both periods; $95.83 \%$ of them did not reach energy requirements and 85.41 protein requirements due to fasting, low acceptance of hospital diet and hospital discharge. The statistical analysis of anthropometric data showed significant difference between adequacy of current body weight/ideal body weight $(\mathrm{p}=0.01)$ in the pre-transplant group as well adequacy of tricipital skin-fold in the post-transplant group $(\mathrm{p}=0.03)$. Conclusion: The nutritional requirements might have been overestimated.

Key words: Nutritional assessment, Nutritional status, Influence, Nutritional intake, Liver transplant. 


\section{REFERÊNCIAS}

1. Küss R, Bourget P. El hígado, el páncreas y el intestino. In Küss R, Bourget $\mathrm{P}$ (eds). Una historia ilustrada del trasplante de órganos. Rueil-Malmaison, Sandoz, 1992; 76-90.

2. Italian Multicentre Cooperative Project on Nutrition in Liver Cirrhosis. Nutritional status in cirrhosis. J hepatology 1994; 21:317.

3. Morgan MY, Levine JA. Nutrition 1996; 12 (6): 430-435.

4. Reilly JJ, Hull SF, Albert N, Waller A et al.JPEN,1998; 12 (4): 371-376.

5. Chima SC, Barco k, Dewitt ML et al.J Am Diet Assoc, 1997; 97 (9): 975-980.

6. Muller MJ.J hepatology 1995; 23 (suppl 1): 31.

7. McCullough AJ, Tavvil AS. Semin Liver Dis 1991; 11:265.
8. Waitzberg DL, Caiaffa WT, Correia MITD. Nutrition 2001; 17: 573-580.

9. Muller MJ, Lautz Hu, Plogmann B, et al. Hepatology 1992; 15:782.

10. Coutinho W: Consenso Latino Americano de obesidade. Arq Bras Endocrinol 43:21, 1999, pg 31.

11. Shoronts E.P. Nutritional assessment of adults with end stage hepatic failure. Nutr Clin Pract 3:113, 1988.

12. Mahan LK, Arlin MT: Alimentos, nutricão e dietoterapia. $9^{a}$ Ed. São Paulo: Roca; 1998, p 333.

13. Krom RAF, Wiesnir RH, Retke SR, et al. Mayo Clinic Proc, 1989; 64; 84-94.

14. Pujol A. Gastroenterology Hepatol 1992; 15: 157-159.

15. Munoz SJ: Semin Liv Dis, 1991; $11: 278$.

Prografß (Tacrolimo) Forma Farmacêutica e Apresentações: Embalagem contendo 100 cápsulas de $1 \mathrm{mg}$. Embalagem contendo 50 cápsulas de $5 \mathrm{mg}$. Solução injetável estéril concentrada em embalagem contendo 10 ampolas de $1 \mathrm{~mL}$ (cada $\mathrm{mL}$ de solução injetável contém $5 \mathrm{mg}$ de Tacrolimo). Uso Adulto e Pediátrico. Indicações e Posologia: Prograf é indicado para a profilaxia da rejeição de órgãos em pacientes que sofreram transplantes alogênicos de fígado e rins. É recomendado que Prograf seja utilizado concomitantemente com corticosteróides adrenais. Prograf SOLUÇÃO INJETÁVEL (Somente para infusão intravenosa): por causa do risco de anafilaxia deve ser reservado aos pacientes que não estão aptos a tomar Prograf cápsulas. A dose inicial de Prograf não deve ser administrada antes de 6 horas depois do transplante e a dose inicial é $0,03-0,05 \mathrm{mg} / \mathrm{kg} /$ dia em forma de infusão EV. Os pacientes adultos devem receber os limites inferiores da faixa de dose. A infusão EV contínua de Prograf solucão injetável deve ocorrer somente até o paciente conseguir tolerar a administracão oral de Prograf cápsulas. Prograf CÁPSULAS: Resumo das recomendacõos de dose oral inicial e as concentracões no sangue total respectivamente: Adultos - Transplante renal: $0.2 \mathrm{mg} / \mathrm{kg} \mathrm{dia}^{*}$, mês 1-3:7-20 ng/mL mês 4 - 12.5-15 ng/mL. Adultos - Transplante hepático: 0.10 - 0.15 mg/kg/dia*, mês 1 -12: 5-20 ng/mL Crianças - Transplante hepático: $0,15-0,20 \mathrm{mg} / \mathrm{kg} / \mathrm{dia} a^{*}$, mês 1 - 12:5-20 ng/mL. * Nota: dividida em duas doses, administradas a cada 12 horas. Transplantes Hepáticos: iniciar terapia oral com Prograf cápsulas se possível. Se a terapia EV for necessária, a mudança de Progra solução injetável para cápsulas é recomendada assim que a terapia oral puder ser tolerada. Em um paciente recebendo infusão intravenosa, a primeira dose da terapia oral deve ser administrada de 8-12 horas depois da descontinuaç̌ăo da infusão intravenosa. Em pacientes receptores de transplante hepático, a administração concomitante com suco de "grape fruit" aumenta as concentracões mínimas de Tacrolimo no sangue. A dose deve ser titulada com base na avaliação clínica de rejeicãa e tolerabilidade. Doses menores de Prograf podem ser suficientes como terapia de manutenção. Transplantes Renais: A dose inicial de Prograf pode ser administrada 24 horas depois do transplante, mas deve ser adiada até a funcão renal se recuperar (como indicado por exemplo pela creatinina sérica $4 \mathrm{mg} / \mathrm{dL}$ ). Pacientes negros podem requerer doses mais elevadas para alcançar concentrações sangüíneas comparáveis. Verificar recomendações sobre as diferenças de doses e concentracõos mínimas entre caucasianos e negros na bula completa. Pacientes Pediátricos: Pacientes pediátricos receptores de transplante hepático sem disfunçãa renal ou hepática pré-existente requereram e toleraram doses mais elevadas que os adultos para alcançr concentracões sangüíneas similares. Portanto, é recomendado que a terapia seja iniciada em pacientes pediátricos com uma dose intravenosa inicial de $0,03-0,05 \mathrm{mg} / \mathrm{kg} / \mathrm{dia}$ e uma dose oral inicial de 0,15-0,20 mg/kg/dia. Ajustes na dose podem ser necessários. A experiência em pacientes pediátricos receptores de transplante hepático é limitada. Pacientes com Disfuncão Renal ou Hepática: devido ao potencial de nefrotoxicidade pacientes com disfunç̧ão renal ou hepática devem receber doses no limite inferior das faixas de dose intravenosa e oral recomendadas. Reducōos adicionais na dose abaixo dessas faixas podem ser necessárias. A terapia de Prograt usualmente deve ser adiada em até 48 horas ou mais em pacientes com oligúria pós-operatória. Conversão de um Tratamento Imunossupressivo para Outro: Prograf não deve ser usado simultaneamente com ciclosporina Prograf our ciclosporina devem ser descontinuados no mínimo 24 horas antes de iniciar o outro. Na presença de concentrações elevadas de Prograf ou ciclosporina, a administração do medicamento usualmente deve ser adiada. CONTRA-INDICAÇÕES: Prograf é contra-indicado descontinuados no mínimo 24 horas antes de iniciar o outro. Na presença de concentrações elevadas de Prograf ou ciclosporina, a administração do medicamento usualmente deve ser adiada. CONTRA-INDICAÇOES: Prograf é contra-indicado desenvolvimento de linfoma podem ser resultado da imunossupressão. Prograf pode. Ausar neurotoxicidade e nefrotoxicidade, particularmente quando usado em doses elevadas. Deve-se tomar cuidado ao utilizar Tacrolimo o possivel desenvolvimento de linfoma podem ser resultado da imunossupressão. Prograf pode causar neurotoxicidade e nefrotoxicidade, particularmente quando usado em doses elevadas. Deve-se tomar cuidado ao utilizar Tacrolimo com outros medicamentos nefrotóxicos. Hiperpotassemia leve a grave foi relatada. Os níveis séricos de potássio devem ser monitorados e diuréticos poupadores de potássio não devem ser utilizados durante a terapia com Prograf. Neurotoxicidade, incluindo tremores, dores de cabeça, e outras alteraçōes na função motora, no nivel mental, e nas funçoes sensoriais foram relatadas. Como em pacientes recebendo outros imunossupressores, pacientes recebendo Prograf tiveram um risco aumentado de desenvolver linfomas e outras doenças malignas, particularmente da pele. O risco parece estar relacionado à intensidade e duração da imunossupressão ao invés de estar relacionado à utilização de algum agente especifico. Um distúrbio linfoproliferativo relacionado à infecçãa pelo virus Epstein-Barr (EBV) foi relatado em receptores de orgãos transplantados imunossuprimidos. O risco de disturbio linfoproliferativo e maior em crianças mais novas que estão sob o risco da infecção primária por EBV enquanto estão imunossuprimidas ou que passam a receber Prograf após um longo período de terapia de imunossupressão. Pacientes recebendo Prograf injetável devem ficar sob observação contínua durante pelos menos 30 minutos após o início da infusão e em intervalos freqüentes após esse período. Se sinais ou sintomas de anafilaxia ocorrerem, a infusão deve ser interrompida. Uma solução aquosa de epinefrina e uma fonte de oxigênio devem estar disponiveis próximas ao leito. PRECAUCCÕES: Gerais: hipertensão. Pacientes com Disfunção Renal e Hepática: devem ser utilizadas doses menores. Hipertrofia do miocárdio: parece ser reversivel na maioria dos casos após a redução da dose ou descontinuacão da terapia. Gravidez e Lactação: em ratos e coelhos, efeitos adversos foram observados nos fetos principalmente em doses elevadas que foram tóxicas para as fêmeas. Não existem estudos adequados e bem controlados em muheres grávidas. TACROLIMO é transferido através da placenta. O uso de Tacrolimo durante a gravidez foi associado com hiperpotassemia neonatal e disfunção renal. Prograf deve ser usado durante a gravidez somente se o benefício para a mãe justificar o risco potencial ao feto. Uma vez que o Tacrolimo é excretado no leite humano, a amamentação durante o tratamento deve ser evitada. Interações Medicamentosas: estudos de interação medicamentosa com Tacrolimo não foram conduzidos. Deve-se tomar cuidado ao administrar Prograf com medicamentos que podem estar relacionados com disfunções renais. Drogas que podem alterar as concentraçoes de Tacrolimo: Drogas que podem aumentar as concentrações de ( Cetoconazol), Agentes gastrin-testinais pro-cineticos.(Cisaprida, Metoclopramida), Outras drogas (Bromocriptina, Cimetidina, Ciclosporina, Danazol, Metiliprednisolona, Inibidores de protease). Drogas que podem diminuir a concentracãa de

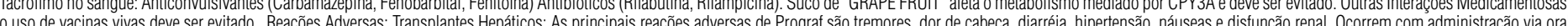
0 uso de vacinas vivas deve ser evilado. Reaçoes Adversas. Transplantes Hepálicos. As principais reaçoes adversas de Prograf são tremores, dor de cabeça, diarrela, hipertensaa, nauseas e disflunção renal. Ocorrem com administração via oral recendo

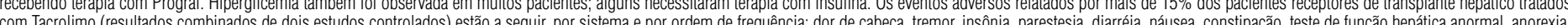
vômito, hiperteñ̃̃ funcão tromer

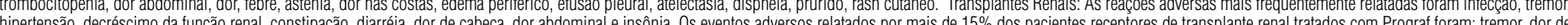
hiper cabeça, ino

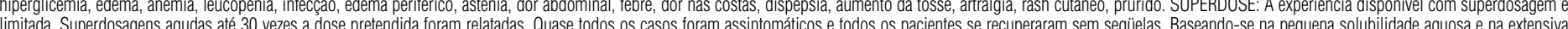

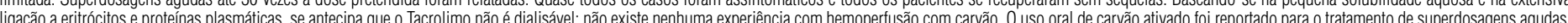

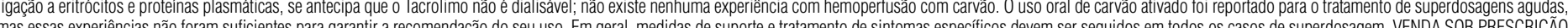

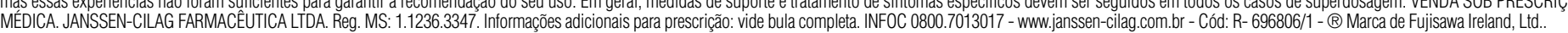

